\title{
MODULAR CONGRUENCES AND THE BROWN-MCCOY RADICAL FOR SEMIGROUPS
}

\author{
D. R. LATORRE
}

\begin{abstract}
The Brown-McCoy radical $R_{G 0}$ for semigroups with zero is characterized in terms of modular two-sided congruences. The general notion of the $\mathcal{C}$-radical of a semigroup is used to prove that $R_{G 0}$ is the $\rho_{s}$-class containing zero, where $\rho_{z}$ is the intersection of all modular maximal two-sided congruences of $S$. Thus when $\rho_{s}$ is the identity relation, $R_{G 0}=0$ and $S$ is isomorphic to a subdirect product of congruence-free semigroups with zero and identity. We also link $R_{G 0}$ to representation theory.
\end{abstract}

1. Introduction. One of the early generalizations of the Jacobson radical, the Brown-McCoy radical [2], offers the advantage that simple rings, instead of dense rings of linear transformations, appear in the subdirect sum representation of a semisimple ring. Moreover, Sulinski [12] has shown the Brown-McCoy radical property to be essentially the smallest radical property for which every semisimple ring is isomorphic to a subdirect sum of simple rings.

The Brown-McCoy radical was studied for categories by Sulinski [13] and for semirings by the present author [7]. Recently, Hoehnke [4] has considered this radical for algebras and its application to semigroups, particularly to semigroups with zero.

Radical theory for semigroups has taken its motivation largely from the Jacobson radical for rings and, because this radical can be described as the intersection of all modular maximal right ideals, various semigroup radicals have effectively used modular maximal right congruences (see references [5], [6], [8], [10], and [11]). However, the Brown-McCoy ring radical can similarly be characterized as the intersection of all modular maximal two-sided ideals. Thus the purpose of this article is to present an alternate approach to the Brown-McCoy radical for semigroups with zero, based on the notion of the $\mathfrak{C}$-radical of a semigroup due to Tully [3, paragraph 11.6], and thereby relate this radical to modular maximal two-sided congruences. These congruences have already been considered in some detail by Oehmke [9] and Arendt [1] with regard to radical theory for semigroups.

Received by the editors August 11, 1970 and, in revised form, November 23, 1970.

AMS 1970 subject classifications. Primary 20M10.

Key words and phrases. Brown-McCoy radical, congruence-free semigroups, modular congruences, radical theory for semigroups.

Copyright (C) 1971. American Mathematical Society 
In $\$ 4$ we characterize the Brown-McCoy radical for a semigroup $S$ with zero as the $\rho_{s}$-class containing zero, where $\rho_{s}$ denotes the intersection of all modular maximal two-sided congruences on $S$. Therefore $\rho_{s}$ is the identity relation on $S$ if and only if $S$ is isomorphic to a subdirect product of congruence-free semigroups with identity, and in this case the radical is zero. Oehmke, and later, Arendt, loc. cit., have further characterized the finite semigroups and bands, respectively, for which $\rho_{s}$ is the identity relation. We also determine a connection between this radical and representation theory in $\$ 5$.

2. Preliminaries. By a type of semigroups we mean a class $\mathfrak{e}$ of semigroups satisfying (i) if $S \in \mathcal{C}$ and $S$ is isomorphic to $S^{\prime}$ then $S^{\prime} \in \mathbb{e}$, and (ii) any semigroup $S$ with $|S|=1$ is in $\mathfrak{e}$.

For any semigroup $S$ and any type $\mathcal{C}$ let $\mathfrak{C}_{s}$ denote the set of all congruences $\sigma$ on $S$ of type $\mathcal{e}$, i.e., all $\sigma$ such that $S / \sigma \in \mathcal{C}$. The $\mathcal{C}$ radical of $S$ is defined by $\mathcal{C}$-rad $S=\bigcap\left\{\sigma: \sigma \in \mathcal{C}_{s}\right\}$.

We henceforth write $\rho_{s}$ for $\mathfrak{C}$-rad $S$. The derived type $\mathfrak{C}^{\prime}$ of $\mathfrak{C}$ is the class of all semigroups $S$ such that $\rho_{s}=\iota$, the identity relation on $S$. If $S \in \mathfrak{C}^{\prime}$ we say that $S$ is $\mathfrak{C}$-radical-free. Always $\mathfrak{C} \subseteq \mathfrak{C}^{\prime}$.

Now let $\mathfrak{C}$ be a type of semigroups such that every $S \in \mathcal{C}$ has a zero element. For each $\sigma \in \mathcal{C}_{s}$ let $z_{\sigma}$ denote the zero of $S / \sigma$, and define $R_{\mathrm{s}}=R_{\mathrm{s}}(\mathfrak{C})=\bigcap\left\{z_{\sigma}: \sigma \in \mathfrak{C}_{\mathrm{s}}\right\}$.

Since each $z_{\sigma}$ is an ideal of $S$, either $R_{8}$ is empty or is itself an ideal of $S$. If $R_{8}$ is not empty it is, of course, the unique $\rho_{\mathrm{s}}$-class that is an ideal of $S$. Thus if $S$ is e-radical-free and $R_{s}$ is not empty, then $S$ has a zero and $R_{s}=\{0\}$.

A two-sided congruence $\sigma$ on $S$ is called modular with respect to an element $a \in S$ provided the $\sigma$-class of $a$ is the identity of $S / \sigma$. We define an element $a \in S$ to be $\mathcal{C}$-regular if the only congruence on $S$ of type $\mathfrak{C}$ which is modular with respect to $a$ is the universal relation $\omega$ on $S$. A subset $X$ of $S$ is called e-regular if every element of $X$ is $\mathcal{C}$ regular.

TheOREM 1. For any semigroup $S$ either $R_{\mathrm{a}}$ is empty or $R_{\mathrm{s}}$ is a Cregular ideal of $S$.

Proof. If $a \in R_{s}$ and $a$ is not $\mathcal{C}$-regular then there is a congruence $\tau \neq \omega$ of type $\mathcal{C}$ on $S$ modular with respect to $a$. Since $a \in R_{s}$ $=\bigcap\left\{z_{\sigma}: \sigma \in \mathcal{C}_{s}\right\}$ we have $a \in z_{\tau}$, whence the $\tau$-class of $a$ is the zero of $S / \tau$. Thus $|S / \tau|=1$, contrary to $\tau \neq \omega$.

In $\S 3$ we characterize $R_{s}$, for a more restricted type $\mathcal{C}$, as the greatest e-regular ideal.

Now define $\bar{R}_{s}$ to be the set of all $x \in S$ for which the ideal $(x)$ of $S$ generated by $x$ is $\mathrm{C}$-regular. 
THEOREM 2. $\bar{R}_{s}$, if nonempty, is a e-regular ideal of $S$ which contains every e-regular ideal of $S$. In particular, $R_{s} \subseteq \bar{R}_{\mathbf{s}}$.

3. Particularly simple semigroups. A semigroup $S$ is said to be congruence-free if it has no two-sided congruences except $\iota$ and $\omega$. A congruence $\sigma$ on a semigroup $S$ will be called maximal if $\sigma=\omega$ or else $\sigma \neq \omega$ and there is no congruence strictly between $\sigma$ and $\omega$. We need the following well-known result.

TheOREM 3. A congruence $\sigma$ on $S$ is maximal if and only if $S / \sigma$ is congruence-free.

We call a congruence-free semigroup having a zero and identity element particularly simple. The class of all particularly simple semigroups is a type of semigroup, and for the remainder of this paper we denote this type by $\mathfrak{C}$. The following are immediate.

Theorem 4. A congruence $\sigma$ on $S$ belongs to $\mathcal{C}_{8}$ iff $\sigma$ is a modular maximal congruence such that $S / \sigma$ has a zero.

ThEOREM 5. Let $S$ be a particularly simple semigroup, $|S|>1$. Then

(a) the identity e of $S$ is not e-regular,

(b) $e \notin R_{s}$,

(c) $S$ is e-radical free,

(d) $R_{\mathrm{s}}=\{0\}$,

(e) $S$ is a 0-simple semigroup.

For completeness we state:

Theorem 6 [3, Theorem 11.23]. If $S$ is any semigroup, $S / \rho_{\mathrm{s}}$ is $\mathrm{e}$ radical-free.

Theorem 7 [3, Exercise 2 (a), P. 278]. A semigroup $S$ is eradical-free if and only if it is isomorphic to a subdirect product of particularly simple semigroups.

Theorem 2 shows that for any type $\mathfrak{C}$ of semigroups, always $R_{\mathfrak{s}} \subseteq \bar{R}_{\mathbf{s}}$. We now prove that for the type $\mathcal{C}$ of particularly simple semigroups, $R_{\mathrm{s}}=\bar{R}_{\mathrm{s}}$.

THEOREM 8. Let $\mathfrak{C}$ be the type of all particularly simple semigroups. For any semigroup $S, R_{s}=\bar{R}_{\mathrm{s}}$.

Proof. Let $b \in \bar{R}_{\boldsymbol{z}}$ and $\sigma \in \mathcal{C}_{\mathrm{s}}$. Consider the ideal $\left(b_{\sigma}\right)$ in $S / \sigma$ generated by $b_{\sigma} \in S / \sigma$. Now $\left(b_{\sigma}\right)=(b) \nu$, where $\nu: S \rightarrow S / \sigma$ is the natural mapping. If $x \in\left(b_{\sigma}\right)$, say $x=y \nu$ with $y \in(b)$, then $y$ is e-regular in $S$. It follows easily that $x$ is e-regular in $S / \sigma$, whence $\left(b_{\sigma}\right)$ is e-regular. 
Thus $b_{\sigma} \in \bar{R}_{S / \sigma}$. But $\bar{R}_{S / \sigma}=\left\{z_{\sigma}\right\}$. For if $a_{\sigma}(a \in S)$ is in $\bar{R}_{S / \sigma}$ then $\left(a_{\sigma}\right)$ is a e-regular ideal of $S / \sigma$, so that $\left(a_{\sigma}\right)=\left\{z_{\sigma}\right\}$ or $\left(a_{\sigma}\right)=S / \sigma$ since $S / \sigma$ is 0 -simple if $\sigma \neq \omega$. If $\left(a_{\sigma}\right) \neq\left\{z_{\sigma}\right\}$ then $\sigma \neq \omega$ and the identity of $S / \sigma$ is C-regular, contrary to Theorem 5. Thus $\left(a_{\sigma}\right)=\left\{z_{\sigma}\right\}$ and $\bar{R}_{S / \sigma}=\left\{z_{\sigma}\right\}$. From $b_{\sigma}=z_{\sigma}$ we have $b \in \cap\left\{z_{\sigma}: \sigma \in \mathcal{C}_{s}\right\}=R_{s}$.

4. The Brown-McCoy radical. The Brown-McCoy radical for semigroups [4], as for rings [2], was developed as a special case of more general radicals. Before linking this semigroup radical with the results of $\$ \$ 1-3$, we indicate its definition, extracting from [4].

For any semigroup $S$ let $C(S)$ denote the lattice of all congruences on $S$ and let $G$ be the mapping $G: S \rightarrow C(S)$ defined as follows: for each $a \in S, G(a)$ is the (two-sided) congruence on $S$ generated by the relation $X_{a}=\{(a x, x): x \in S\}$ on $S$.

For each congruence $\sigma$ on $S$, define

$$
\begin{aligned}
\sigma^{0} & =\varnothing, \text { if } S / \sigma \text { has no zero; } \\
& =\text { the zero element } z_{\sigma} \text { of } S / \sigma, \text { if } S / \sigma \text { has a zero. }
\end{aligned}
$$

Since for each $a \in S, G(a) \in C(S)$, we consider $G(a)^{0}$. Let $G^{0}(a)=G(a)^{0}$. The element $a \in S$ is said to be $G^{0}$-regular if $a \in G^{0}(a)$. Let $a^{(0)}$ denote the intersection of all $\sigma^{0}$ that contain $a \in S$. The Brown-McCoy radical $R_{G^{0}}=R_{G^{0}}(S)$ of $S$ is defined to be the set $R_{G^{0}}=R_{G^{0}}(S)$ $=\left\{a \in S: a^{(0)}\right.$ is $G^{0}$-regular $\}$.

Now for any semigroup $S$ with zero, and any $\sigma \in C(S)$, we have $\sigma^{0}=\{x \in S:(x, 0) \in \sigma\}$. Thus $0^{(0)}=\{0\}$, and 0 is $G^{0}$-regular so 0 $\in R_{G^{0}}(S)$. Clearly $a$ is $G^{0}$-regular provided $(a, 0) \in G(a)$. An explicit characterization for $G(a)$ is given in [4].

The above notion of $G^{0}$-regularity coincides with that of $G$-regularity for rings used in the definition of the Brown-McCoy ring radical. (An element $a$ in a ring $R$ is $G$-regular if $a$ belongs to the ideal of $R$ generated by the set $\{a x+x: x \in R\}$; see [2].) However, it can be shown that an element $a$ in ring $R$ is $G$-regular if and only if the only maximal congruence on $R$ modular with respect to $a$ is the universal relation. We are thus led to formulate the following theorem.

TheOREM 9. Let $S$ be a semigroup with zero. An element $a \in S$ is $G^{0}$ regular if and only if the only maximal congruence on $S$ modular with respect to $a$ is the universal relation.

In the terminology of the preceding section: An element $a \in S$ is $G^{0}$ regular if and only if $a$ is $\mathrm{C}-r e g u l a r$, where $\mathfrak{C}$ is the type of particularly simple semigroups. 
Proof. Suppose $\sigma$ is a proper maximal congruence on $S$ modular with respect to $a$. Then $X_{a}=\{(a x, x): x \in S\} \subseteq \sigma$, so that $G(a) \subseteq \sigma$. If $a$ were $G^{0}$-regular then $(a, 0) \in G(a) \subseteq \sigma$, whence $a_{\sigma}$ is both the zero and identity of $S / \sigma$, contrary to $\sigma \neq \omega$. Thus $a$ is not $G^{0}$-regular.

For the converse, suppose $a$ is not $G^{0}$-regular, i.e. $(a, 0) \notin G(a)$. Using Zorn's lemma, let $\alpha$ be a congruence on $S$ maximal in the class of all congruences on $S$ that contain $G(a)$ but not $(a, 0)$. Now $\alpha$ is a proper maximal congruence on $S$. For if $\beta \in C(S)$ and $\alpha \subset \beta$ then $(a, 0) \in \beta$. Let $x_{\beta}$ denote the $\beta$-class containing any $x \in S$. Since $X_{a} \subseteq G(a) \subseteq \alpha \subseteq \beta$ we have $x_{\beta}=(a x)_{\beta}=a_{\beta} x_{\beta}=0_{\beta} x_{\beta}=0_{\beta}$ for every $x \in S$, and so $\beta=\omega$ and $\alpha$ is maximal. Because $\alpha$ is maximal, $S / \alpha$ is congruence-free, and $a_{\alpha}$ is a left identity for $\alpha$ since $X_{a} \subseteq \alpha$. But any onesided identity of a congruence-free semigroup with zero is necessarily a two-sided identity, so $\alpha$ is therefore a proper maximal congruence modular with respect to $a$.

We now give the main result of this work.

THEOREM 10. Let $\mathfrak{C}$ be the type of all particularly simple semigroups. For any semigroup $S$ with zero, $R_{8}=\bar{R}_{s}=R_{G^{0}}(S)$.

Proof. By Theorem 3.4 of [4], $R_{G^{0}}(S)$ is an ideal of $S$, and $R_{G^{0}}(S)$ is by definition $G^{0}$-regular. Thus by Theorem $9, R_{G^{0}}(S)$ is a C-regular ideal. Since $R_{s}=\bar{R}_{s}$ is the greatest $\mathcal{C}$-regular ideal of $S$, we have $R_{G^{0}}(S) \subseteq R_{s}=\bar{R}_{s}$.

Conversely, if $a \in S$ and $a \notin R_{G^{0}}(S)$ then $a^{(0)}$ is not $G^{0}$-regular. Thus let $e \in a^{(0)}$ such that $e$ is not $G^{0}$-regular. By Theorem 9 , there is a proper maximal congruence $\sigma$ on $S$ modular with respect to $e$. Since $\sigma \neq \omega, e \notin z_{\sigma}$. But then $a \notin z_{\sigma}=\sigma^{0}$, as otherwise $e \in a^{(0)} \subseteq \sigma^{0}=z_{\sigma}$. Since $a \notin z_{\sigma}$ and $\sigma \in \mathcal{C}_{8}$, certainly $a \notin R_{s}=\bigcap\left\{z_{\sigma}: \sigma \in \mathcal{C}_{s}\right\}$.

Theorem 10 shows that the Brown-McCoy radical $R_{G^{0}}(S)$ is the $\rho_{s}$-class which contains zero (alias $R_{8}$ ), where $\rho_{s}$ is the intersection of all modular maximal congruences on $S$. By Theorem $7, \rho_{s}=\iota$ if and only if $S$ is isomorphic to a subdirect product of particularly simple semigroups; in this case $R_{G^{0}}(S)=0$. Oehmke [9, Theorem 28] has further characterized the finite semigroups for which $\rho_{s}=\iota$, and Arendt [1, Theorem 16] has done the same for bands.

5. Relation to representation theory. By a representation of a semigroup $S$ (by transformations of a set $M$ ) we mean a homomorphism $\phi$ of $S$ in to the full transformation semigroup $T_{M}$ on $M$. For the associated notion of a (right) operand (or $S$-system), and the more special notion of an operator equivalence on an operand, we refer to $[3$, Chapter 11] or [6]. 
If $S$ is a semigroup, we denote by $S_{8}$ the operand over $S$ associated with the regular representation of $S$, i.e., the operand in which $S$ acts on itself by right multiplication. The operator equivalences on $S$, are precisely the right congruences on the semigroup $S$. For any right congruence $\sigma$ on $S$ we denote the operand $S_{s} / \sigma$ by $S / \sigma$; it consists of all the $\sigma$-classes $x_{\sigma}$ of $S_{s}$, with the product of $x_{\sigma}$ by any element $a \in S$ defined by $x_{\sigma} a=(x a)_{\sigma}$. We are particularly interested in the case where $\sigma$ is also a two-sided congruence on $S$. In this event, the operand $S / \sigma$ and the factor semigroup $S / \sigma$ are related by $x_{\sigma} a=(x a)_{\sigma}=x_{\sigma} a_{\sigma}$. That is, the product in the operand $S / \sigma$ of $x_{\sigma}$ by $a \in S$ coincides with the product in the semigroup of $x_{\sigma}$ and $a_{\sigma}$. For notational convenience, let $(S / \sigma) a=\left\{x_{\sigma} a: x_{\sigma} \in S / \sigma\right\}$.

Let $R$ be any ring whose Brown-McCoy radical, $G(R)$, is not $R$. If $\left\{M_{\alpha}\right\}_{\alpha \in \Omega}$ denotes the set of all modular maximal two-sided ideals of $R$, it is well known that

$$
G(R)=\bigcap_{\alpha \in \Omega} M_{\alpha}=\bigcap_{\alpha \in \Omega}\left(M_{\alpha}: R\right)=\bigcap_{\alpha \in \Omega}\left(\overline{0}_{\alpha}: R / M_{\alpha}\right),
$$

where $\left(M_{\alpha}: R\right)=\left\{a \in R: R a \subseteq M_{\alpha}\right\}$. Thus $a \in G(R)$ if and only if $\left(R / M_{\alpha}\right) a=\overline{0}_{\alpha}$ for all $\alpha \in \Omega$. Motivated in this way, we proceed as follows.

Let $\mathfrak{C}$ be the type of all particularly simple semigroups. For any semigroup $S$, define

$$
R_{s}^{\prime}=R_{s}^{\prime}(\mathcal{C})=\left\{a \in S:(S / \sigma) a=z_{\sigma} \text { for all } \sigma \in \mathcal{C}_{s}\right\}
$$

If $\mathbb{e}_{s}=\{\omega\}$ then $R_{s}^{\prime}=S$, but $R_{s}^{\prime}$ may be empty otherwise. If $S$ has a zero element 0 , then $0 \in R_{s}^{\prime}$. Our basic result for $R_{s}^{\prime}$ is the following.

THEOREM 11. Let $\mathfrak{C}$ be the type of all particularly simple semigroups. For any semigroup $S$ with zero, $R_{s}^{\prime}=R_{s}=\bar{R}_{s}=R_{G^{0}}(S)$.

Proof. If $a \in R_{s}$ then $a \in z_{\sigma}$ for every $\sigma \in \mathcal{C}_{s}$, so that $a_{\sigma}=z_{\sigma}$ in the semigroup $S / \sigma$. Thus for any $x_{\sigma}$ in the operand $S / \sigma$ we have $x_{\sigma} a$ $=(x a)_{\sigma}=x_{\sigma} a_{\sigma}=x_{\sigma} z_{\sigma}=z_{\sigma}$, whence $(S / \sigma) a=z_{\sigma}$ and $a \in R_{s}^{\prime}$.

Conversely, let $a \in R_{s}^{\prime}$. If $\sigma \in \mathcal{C}_{\text {s }}$ then $(S / \sigma) a=z_{\sigma}$, so that $x_{\sigma} a=z_{\sigma}$ for all $x_{\sigma} \in S / \sigma$. Since $\sigma \in \mathcal{C}_{s}, S / \sigma$ has an identity element $e_{\sigma}(e \in S)$, and $e_{\sigma} a=(e a)_{\sigma}=e_{\sigma} a_{\sigma}=a_{\sigma}$. Thus $a_{\sigma}=z_{\sigma}$ so $a \in z_{\sigma}$. It follows that $a \in R_{\varepsilon}$.

\section{REFERENCES}

1. B. D. Arendt, Semisimple bands, Trans. Amer. Math. Soc. 143 (1969), 133143. MR $40 \# 255$.

2. B. Brown and N. H. McCoy, Radicals and subdirect sums, Amer. J. Math. 69 (1947), 46-58. MR 8, 433. 
3. A. H. Clifford and G. B. Preston, The algebraic theory of semigroups. Vol. II, Math. Surveys, no. 7, Amer. Math. Soc., Providence, R. I., 1967. MR 36 \#1558.

4. H. J. Hoehnke, Das Brown-McCoysche 0-Radikal für Algebren und seine Anwendung in der Theorie der Halbgruppen, Fund. Math. 66 (1969/70), 155-175. MR 40 \#7178.

5. —, Zur Strukturtheorie der Halbgruppen, Math. Nachr. 26 (1963), 1-13. MR $28 \# 4051$.

6. - Structure of semigroups, Canad. J. Math. 18 (1966), 449-491. MR 33 $\# 5762$.

7. D. R. LaTorre, The Brown-McCoy radicals of a hemiring, Publ. Math. Debrecen 14 (1967), 15-28. MR 36 \#3828.

8. - An internal characterization of the 0-radical of a semigroup, Math. Nachr. 45 (1970), 279-281.

9. R. H. Oehmke, On maximal congruences and finite semisimple semigroups, Trans. Amer. Math. Soc. 125 (1966), 223-237. MR 34 \#2739.

10. H. Seidel, Über das Radikal einer Halbgruppe, Math. Nachr. 29 (1965), 255263. MR 32 \#1276.

11. R. E. Slover, Representations of a semigroup, Trans. Amer. Math. Soc. 120 (1965), 417-427. MR 32 \#5765.

12. A. Sulin'skil, Some questions in the general theory of radicals, Mat. Sb. 44 (86) (1958), 273-286. MR 20 \#4581. (Russian)

13. - The Brown-McCoy radical in categories, Fund. Math. 59 (1966), 23-41. MR 34 \#1378.

Clemson University, Clemson, South Carolina 29631 